\title{
Nutritional support in patients with advanced cancer: permission to fall out?
}

\author{
A. W. McKinlay \\ Gastrointestinal Unit, Aberdeen Royal Infirmary, Foresterhill, Aberdeen AB25 2ZN, UK
}

\begin{abstract}
Data from the European Society for Parenteral and Enteral Nutrition Home Artificial Nutrition Survey suggest that the use of home parenteral nutrition (HPN) in patients with cancer as the primary diagnosis varies markedly between different countries in Europe, being highest in The Netherlands and low in the UK. This finding is difficult to explain on the basis of cancer incidence. The main indication for HPN remains malignant gastrointestinal tract obstruction, which occurs most frequently in gynaecological and colon cancers. The use of HPN should be planned in the light of the proposed cancer treatment and should be discussed beforehand with the patient. Before HPN is considered, a patient should typically require intravenous fluids to maintain hydration, be capable of self care, be able to control the treatment, have an expected survival of $\geq 3$ months and have no other available route of feeding. The effect of HPN on quality of life remains controversial, but nutrition is only one of the factors that influence the quality of life in cancer patients. The differing rates of HPN in Europe are probably related to cultural attitudes to incurable cancer as much as to meeting the nutritional needs of the patient.
\end{abstract}

Advanced cancer: Malignant gastrointestinal obstruction: Home parenteral nutrition: Quality of life

Cancer is a common experience in Europe, with an estimated incidence of $2.6 \times 10^{6}$ cases per year (Bray et al. 2002). A number of patients will have primary or secondary disease that impairs the function of their gastrointestinal tract to the extent that they will require nutritional support. Of these patients an even smaller group will have such severe disruption of function that they will require feeding parenterally. Common sense would suggest that the number of patients requiring home parenteral nutrition (HPN) would be constant across Europe. The data are limited and open to criticism, but suggest that the number of patients on HPN varies markedly from country to country, implying that factors other than nutritional need may influence practice.

The European Society for Parenteral and Enteral Nutrition Home Artificial Nutrition Survey collected data from seven European countries in 1997 (Bakker et al. 1999). The prevalence of HPN was found to vary from $3 \cdot 7 / 10^{6}$ cases in the UK to $12 \cdot 7 / 10^{6}$ cases in Denmark. Whilst $5 \%$ of the HPN patients in the UK had cancer as a primary diagnosis, in The Netherlands it was $60 \%$. One explanation for these differences might be that patients in the UK have less access to HPN in general, and are, therefore, less likely to receive nutritional support if they have cancer.
In Scotland, however, all patients receiving HPN are supervised as part of a managed clinical network. The population of Scotland is $5 \cdot 6$ million and sixty-six patients are currently enrolled in the network. Careful inquiry suggests that all patients on HPN in Scotland are accounted for, indicating a prevalence for HPN of $14 / 10^{6}$ cases, which is similar to that reported from Denmark. Only two patients in Scotland are currently receiving HPN for cancer (J Baxter, personal communication). This situation suggests that the low rate of HPN for cancer in the UK is not only related to lack of access to treatment, it may reflect a more fundamental difference in attitude towards nutritional support in malignancy. What factors determine this difference?

\section{Factors that influence the decision for nutritional support}

Decisions relating to nutritional support are not usually reached in isolation, but involve an assessment of the underlying disease, the patient and their wishes, and a nutritional evaluation that defines the specific nutritional needs and requirements. 
It is estimated that $\geq 80 \%$ of patients with malignant disease will suffer weight loss and malnutrition during the course of their illness (Schattner, 2003). Some of this outcome is attributable to failure of intake, but often it is part of a wider cancer-cachexia-anorexia syndrome in which involuntary weight loss, fatigue, anaemia, progressive wasting and tissue depletion occur for multifactorial reasons that are incompletely understood at present. Many metabolic pathways can be altered, including carbohydrate metabolism with increased peripheral insulin resistance, glucose intolerance and high rates of glucose turnover. Fat and protein metabolism may change in ways that lead to a poorer adaptation to the fasting state (Barrera, 2002). It is, therefore, naïve to view nutritional support in patients with cancer as simply an exercise in correcting malnutrition by replacing protein and energy in sufficient quantities to make them gain weight. As Barrera (2002) has suggested, the aim of therapy is usually to minimise weight loss and prevent specific deficiencies until treatment is completed and healing has occurred. In malignant disease the response to anti-cancer treatment is often well defined in statistical terms, but may be difficult to predict for the individual patient. Furthermore, the healing process is often prolonged and can be incomplete, making the time frame for nutritional support difficult to anticipate. For these reasons it is very important that nutrition support teams have a detailed knowledge of the tumour and the treatment plans, particularly where the cancer is in an advanced stage.

\section{The disease}

The management of the underlying disease in patients with advanced malignancy is often complex and involves balancing the treatment of the cancer itself against the need for palliation of symptoms and the requirement for general supportive measures such as nutrition. The wishes of the patient are clearly paramount, but have to be viewed within the overall context of the disease. Given the choice, most patients would like to be cured of their cancer, but this outcome is not always possible.

It is difficult to advise on nutritional support without a thorough assessment of the underlying malignancy and an understanding of the natural history of the disease, the options available for treatment, their chance of success and the burden of treatment on the patient. For these reasons nutritional support should not be initiated in isolation, but should be developed as part of an overall care plan involving the patient and their attending oncologist or surgeon.

On the other hand, what about patients with very advanced disease and uncertain prognoses or those who are 'terminal', many of whom will be severely malnourished? Should they be considered for nutritional support? Bozzetti (2003) has discussed the problems of definition, particularly in relation to expressions like 'terminal', which can be ambiguous. 'All terminal cancer patients are 'oncologically' incurable, [but] not all incurable cancer patients are 'biologically' terminal' (Bozzetti, 2003). The reverse also applies. A patient may have a potentially curable malignancy but still be untreatable and, therefore, effectively 'terminal', because severe concomitant disease makes the risks of the cancer therapy unacceptable. The key factor is clearly the performance of the patient in terms of their tasks of daily living and their quality of life. Performance scores such as that of Karnofsky et al. (1948) have been used for many years by oncologists, but quality of life is more difficult to assess, particularly in individuals with a limited lifespan, and few studies relating to HPN and malignancy have been published (Buchman, 2002).

In considering the underlying disease, therefore, it is important to define the purpose of the therapy. Clearly, if malnutrition is one of the underlying factors that is preventing treatment, then it may need to be addressed. If, however, there are other reasons that make the prospect of intervention untenable, then a more cautious approach to nutritional support may need to be considered. Equally, a patient may have an 'incurable' cancer but still warrant anti-cancer therapy for its palliative effects or to buy time. Nutritional intervention may be entirely appropriate under these circumstances.

Usually, it is an assessment of the patient's nutritional status that determines their need for nutritional support, but in malignancy it is the underlying disease that is the most important driver.

\section{The patient}

If after a careful assessment of the underlying malignancy it is accepted that a patient with cancer should be considered for nutritional support, what other factors should be taken into account?

Clearly, the attitude of the patient is vitally important, as complex nutritional support adds an extra burden at a time when they may already be coming to terms with the underlying diagnosis and coping with effects of radiotherapy, chemotherapy or surgery.

When a patient is able to eat it is often forgotten that food is the best form of nutritional support. Unfortunately, food in hospital is sometimes of poor quality, is not appetising and is presented poorly, which does not encourage a patient with a poor appetite to eat. In the NHS catering departments have often been a 'soft' target for financial savings and are often designated as 'hotel' or 'support' services. Recently, there have been some encouraging signs that the importance of hospital catering has at last been recognised in the UK. The Better Hospital Food Project (Department of Health, 2003) in England has provided practical advice on everything from menus to the provision of food. North of the border, NHS Quality Improvement Scotland, an independent health authority responsible for clinical standards in the Scottish Health Service, has published a standard on hospital food, fluid and nutritional care (NHS Quality Improvement Scotland, 2003). The standard requires Health Boards to have a strategic plan relating to catering, requires hospitals to bring in compulsory screening for undernutrition in patients and sets quality criteria for catering and the delivery of food to the wards. Hopefully, these changes will benefit all patients, but should be particularly relevant to those with malignancy.

It is often assumed that because enteral tube feeding is simple to initiate it is, therefore, unlikely to be detrimental. This premise is not necessarily valid and has been 
questioned in relation to non-cancer patients (Hardy \& Campos, 2003). Nasogastric tubes can be uncomfortable, and patients may experience nausea, vomiting and diarrohea, in addition to any gastrointestinal symptoms directly attributable to the tumour. Enteral tube feeding often fails to deliver the patients prescribed nutritional requirements and does not prevent aspiration. It is, however, easier to teach a patient or carer to use a gastrostomy tube than to handle parenteral nutrition (PN), and the logistics of getting patients home are simpler.

$\mathrm{PN}$ is mainly indicated in patients who no longer have a functional gastrointestinal tract and who are unlikely to recover. In the acute setting nutritional failure can be related to the short-term effects of chemotherapy and radiation, but in the chronic situation the most common indication is probably malignant gastrointestinal obstruction (MGO). Patients with oesophageal and gastric tumours are often more amenable to palliative measures such as stenting or the insertion of a gastrostomy for access (Schattner, 2003). In contrast, patients with intestinal obstruction secondary to carcinoma of the pancreas, ovary or colon can be more difficult to stent, and are more likely to be candidates for PN.

Malignant intestinal obstruction. The pathophysiology of bowel obstruction in patients with cancer is complex and is not always related to the direct infiltration of the bowel by tumour. Obstruction may be attributable to mechanical causes such as adhesions, hernia or radiation effects, and is often exacerbated by drugs such as opiates (Ripamonti \& Bruera, 2002). Benign causes are more common in colo-rectal cancer than in carcinoma of the ovary, where only $6 \%$ of cases are non-malignant (Spears et al. 1988). True MGO as a result of carcinomatosis or direct spread is difficult to manage and can be very unpleasant for the patient. A review of the literature suggests that for ovarian cancer the surgical mortality rate is between 9 and $40 \%$, with a complication rate of $7-90 \%$. Most authors report median survival times of between 2 and 7 months (Ripamonti \& Bruera, 2002).

For these reasons surgical intervention should be undertaken with caution and only after a careful assessment of the risks and benefits. The onset of obstruction in cancer patients is frequently gradual and strangulation is uncommon, so there is usually time to discuss the issues with the individual. Preoperative PN can be helpful, but the same considerations should apply. The benefits should outweigh the risks and some thought needs to be given to the consequences of starting nutritional support. How long will it continue and what will happen if surgery is cancelled or unsuccessful?

The benefits of PN during cancer chemotherapy are also unclear (Klein et al. 1997). A review of eighteen prospective randomised controlled trials (Klein \& Koretz, 1994) found no advantage for survival or reduction in treatment toxicity, although none addressed performance or quality of life issues. In summary, therefore, there is currently little evidence to justify the use of PN routinely in all patients with MGO on grounds of efficacy alone.

There are, however, potential adverse effects of PN, most notably the risk of infection, which has been reported to be higher in patients with cancers receiving PN than in controls (Klein \& Koretz, 1994). A more recent trial of peri-operative feeding in which only malnourished patients with cancer were randomised has reported a lower overall rate of infectious complications in the $\mathrm{PN}$ group than in controls, suggesting that newer techniques and careful attention to detail may overturn some previous assumptions (Bozzetti et al. 2000). Despite this finding, however, it is clear that PN should not be viewed as a routine treatment for all oncology patients. The maximum benefits are derived in patients who are malnourished and for whom there are defined therapeutic options such as surgery.

\section{Nutritional needs}

If it is acknowledged that many of the decisions relating to nutrition are subsidiary to the overall cancer strategy, then what role is left for the nutrition team to play? The very complexity of cancer treatment dictates that any nutritional support is best delivered by a multi-professional team with experience in prescribing and with the expertise to assess and access the gut as well as to deliver intravenous feeding safely. If HPN is to be considered, then logic dictates that the patient should be looked after by a nutrition team that is experienced in the provision of HPN and is within a reasonable travelling distance for the patient. Help should be easily accessible either by telephone or if required by admission to a defined Unit within the hospital.

The assessment of the patient should obviously address their requirements, including the provision of adequate energy and $\mathrm{N}$, but overfeeding should be avoided. This approach, of course, requires some allowance for the patient's oral intake, which initially may be normal, but which may also become negligible in patients with MGO. Patients with cancer should be weighed if active support is being considered, but some will be oedematous or have ascites, for which allowance must be made. Similarly, patients with cancer may have marked micronutrient deficiencies, with consequent implications for wound healing and their response to injury.

There has rightly been renewed debate about the complication rate from enteral tube feeding and PN, and it is clear that parenteral feeding can be delivered safely to many patients in hospital. However, this procedure is different from discharging a patient and providing HPN, for which a certain amount of training or nursing support will be required and organising the logistics of delivery and storage may take time. Time is one commodity that patients with advanced malignancy may not possess. Only $15 \%$ of patients with disseminated tumours survive $>1$ year on HPN, and in one study the median survival time for patients with gynaecological malignancies was reported to be $72 \mathrm{~d}$ and for non-gynaecological tumours $52 \mathrm{~d}$ (King et al. 1993). If enteral tube feeding is possible, and well tolerated, then it may prove an easier solution in patients with cancer. In those patients unfortunate enough to have developed MGO, HPN will be required, but its effect on quality of life remains controversial.

Buchman (2002) argues that factors other than nutrition may determine the quality of life in advanced cancer; for example, the patients' overall feeling of well-being, their actual physical well-being and their ability to control their 
symptoms. Additionally, the loss of the ability to eat is often a negative factor.

A recent Italian multicentre study has evaluated quality of life in sixty-nine adult patients with cancer, most with MGO, all of whom were given HPN (Bozzetti et al. 2002). The median duration for HPN was reported to be 4 months and all patients maintained the same nutritional status until death, which is a considerable achievement. Most patients reported themselves as 'well' despite more than half having worries, desperate feelings about the future, tension and being unable to work, walk outside, do housekeeping or climb stairs. Overall, quality of life scores improved in approximately $40 \%$, and deteriorated in $50 \%$, with scores stabilising over the first month and deteriorating over the last 2 months of life. It would appear, therefore, that to derive benefit from HPN patients should be expected to survive for a minimum of 3 months. The median survival time was 4 months, suggesting that improvements in quality of life are relatively short. Whilst the lack of a control group is understandable, it makes assessment of the duration and real effect on quality of life more difficult to assess.

What is striking about the Italian study is that of sixtynine patients only thirty were aware of their cancer diagnosis and only six were fully aware of their prognosis. This finding raises questions about informed consent, although the authors have argued that the amount of information a patient is given before death depends on their ability to deal with the possibility of imminent death, and that gradual, rather than abrupt, disclosure is the best policy. This approach certainly seems at odds with the present author's perception of practice in the UK, where the trend seems to be towards 'complete' rather than 'abrupt' disclosure. Perhaps, however, it provides an important insight into the variations in the use of HPN in advanced cancer. HPN is only indicated in a small subgroup of patients who do not have functional gastrointestinal tracts, most of whom will have MGO. In the majority of cancers, patients can eat or access their gastrointestinal tract, and the need for HPN doesn't arise. The use of HPN is, therefore, a poor marker for the efficiency or availability of either specialist cancer or nutrition services. Its use depends on the attending medical team's perception of the patient's prognosis, quality of life and attitude to death. In the absence of either a good evidence base for quality of life, or universally-accepted guidelines, the use of HPN in advanced malignancy probably says more about a country's culture or attitudes to palliation than about medical judgement.

\section{Conclusions}

At present there appears to be little choice between two extreme views. On the one hand, there seems to be a model that promotes full disclosure of information relating to the disease and its prognosis, with a desire to avoid false hope and to let the patient face the inevitable without the encumbrance of technology and the risk of complications. Many of these sentiments are worthy and certainly fulfill the criterion of avoiding unnecessary harm. There is, however, a risk of depriving some patients of valuable time, of failing to get them home and of missing an opportunity to improve their quality of life, if only for a short period.

On the other hand, there is an approach that favours complex technology, perhaps avoids telling some patients the full truth about their illness and trades modest shortterm improvements in the quality of life of some patients against an unnecessary risk of complications and further hospitalisation in other patients. In the extreme it fails to acknowledge that patients sometimes need 'permission to fall out', and to be allowed to die with dignity.

How then can a middle ground or consensus about complex nutritional techniques in patients with advanced incurable cancer be established? First, there is a clear need for controlled trials. The evidence does not categorically favour one approach or the other. The design of an ethical study would be difficult but not insurmountable.

Without a reliable evidence base it is difficult to construct practical guidelines. Over 20 years ago Weiss et al. (1982) proposed six criteria, recently restated by Buchman (2002):

1. intravenous fluid is required to maintain fluid and nutritional equilibrium;

2. the patient is capable of self care and able to spend $>50 \%$ of the time out of bed;

3 . the patient is able mentally, physically and emotionally to start, stop and control the HPN infusion;

4. the patient's expected survival is $\geq 3$ months;

5. the patient is aware of their diagnosis and desires HPN;

6. there is no alternative to parenteral feeding in order to provide nutrition.

Not every patient needs to meet all the statements, but a failure to meet some of the criteria should at least precipitate a careful re-examination of the case.

\section{Acknowledgements}

The author thanks Dr Marianne Nicholson, Consultant Oncologist, NHS Grampian, and members of the Nutrition Team, Aberdeen Royal Infirmary for advice and support.

\section{References}

Bakker H, Bozzetti F, Staun M, Leon-Sanz M, Hebuterne X, Pertkiewicz M, Shaffer J \& Thul P (1999) Home parenteral nutrition in adults: a european multicentre survey in 1997. ESPEN-Home Artificial Nutrition Working Group. Clinical Nutrition 18, 135-140.

Barrera R (2002) Nutritional support in cancer patients. Journal of Parenteral and Enteral Nutrition 26, S63-S71.

Bozzetti F (2003) Home total parenteral nutrition in incurable cancer patients: a therapy, a basic humane care or something in between? Clinical Nutrition 22, 109-111.

Bozzetti F, Cozzaglio L, Biganzoli E, Chiavenna G, De Cicco M, Donati D, Gilli G, Percolla S \& Pironi L (2002) Quality of life and length of survival in advanced cancer patients on home parenteral nutrition. Clinical Nutrition 21, 281-288.

Bozzetti F, Gavazzi C, Miceli R, Rossi N, Mariani L, Cozzaglio L, Bonfanti G \& Piacenza S (2000) Perioperative total parenteral nutrition in malnourished, gastrointestinal cancer patients: a randomized, clinical trial. Journal of Parenteral and Enteral Nutrition 24, 7-14. 
Bray F, Sankila R, Ferlay J \& Parkin DM (2002) Estimates of cancer incidence and mortality in Europe in 1995. European Journal of Cancer 38, 99-166.

Buchman AL (2002) Must every cancer patient die with a central venous catheter? (comment). Clinical Nutrition 21, 269-271.

Department of Health (2003) Better hospital food. http://patient experience.nhsestates.gov.uk/bhf/bhf_content/home/home.asp

Hardy G \& Campos A (2003) Editorial comment: if patients prefer parenteral nutrition; is enteral nutrition failing to deliver? Current Opinion in Clinical Nutrition and Metabolic Care 6, 307-311.

Karnofsky DA, Abelman WH, Craver LF \& Burchenal JH (1948) The use of nitrogen mustards in the palliative treatment of carcinoma. Cancer 1, 634.

King LA, Carson LF, Konstantinides N, House MS, Adcock LL, Prem KA, Twiggs LB \& Cerra FB (1993) Outcome assessment of home parenteral nutrition in patients with gynecologic malignancies: what have we learned in a decade of experience? Gynecologic Oncology 51, 377-382.

Klein S, Kinney J, Jeejeebhoy K, Alpers D, Hellerstein M, Murray M \& Twomey P (1997) Nutrition support in clinical practice: review of published data and recommendations for future research directions. Summary of a conference sponsored by the National Institutes of Health, American Society for Parenteral and Enteral Nutrition, and American Society for Clinical Nutrition. American Journal of Clinical Nutrition $\mathbf{6 6}$, 683-706.

Klein S \& Koretz RL (1994) Nutrition support in patients with cancer: what do the data really show? Nutrition in Clinical Practice 9, 91-100.

NHS Quality Improvement Scotland (2003) Standard for Hospital Food, Fluid and Nutritional Care in Hospitals. Edinburgh: NHS QIS.

Ripamonti C \& Bruera E (2002) Palliative management of malignant bowel obstruction. International Journal of Gynecological Cancer 12, 135-143.

Schattner M (2003) Enteral nutritional support of the patient with cancer: route and role. Journal of Clinical Gastroenterology 36, 297-302.

Spears H, Petrelli NJ, Herrera L \& Mittelman A (1988) Treatment of bowel obstruction after operation for colorectal carcinoma. American Journal of Surgery 155, 383-386.

Weiss SM, Worthington PH, Prioleau M \& Rosato FE (1982) Home total parenteral nutrition in cancer patients. Cancer $\mathbf{5 0}$, 1210-1213. 
https://doi.org/10.1079/PNS2004377 Published online by Cambridge University Press 